\title{
Lactation Disorder, CTCAE
}

National Cancer Institute

\section{Source}

National Cancer Institute. Lactation Disorder, CT CAE. NCI Thesaurus. Code C143634.

A disorder characterized by disturbances of milk secretion. It is not necessarily related to pregnancy that is observed in females and can be observed in males. 